\title{
Comparison of Emergency Medical Services Delivery Performance using Maximal Covering Location and Gradual Cover Location Problems
}

\author{
Mohd Hafiz Azizan ${ }^{1}$, Ting Loong Go² , W. A. Lutfi W. M. Hatta ${ }^{3}$, Cheng Siong Lim ${ }^{4}$, \\ Soo Siang Teoh $^{5}$ \\ 1, 2, 3, 4 Faculty of Electrical Engineering, Universiti Teknologi Malaysia, 81310 UTM Skudai, Johor, Malaysia \\ ${ }^{5}$ School of Electrical and Electronic Engineering, Universiti Sains Malaysia, 14300 Nibong Tebal, Malaysia
}

\begin{tabular}{l} 
Article Info \\
\hline Article history: \\
Received Feb 14, 2017 \\
Revised May 30, 2017 \\
Accepted Aug 11, 2017 \\
\hline Keywords: \\
Ambulance location model \\
Gradual cover location problem \\
Maximal coverage location \\
problem \\
Particle swarm optimization
\end{tabular}

\begin{abstract}
Ambulance location is one of the critical factors that determine the efficiency of emergency medical services delivery. Maximal Covering Location Problem is one of the widely used ambulance location models. However, its coverage function is considered unrealistic because of its ability to abruptly change from fully covered to uncovered. On the contrary, Gradual Cover Location Problem coverage is considered more realistic compared to Maximal Cover Location Problem because the coverage decreases over distance. This paper examines the delivery of Emergency Medical Services under the models of Maximal Covering Location Problem and Gradual Cover Location Problem. The results show that the latter model is superior, especially when the Maximal Covering Location Problem has been deemed fully covered.
\end{abstract}

Copyright $\odot 2017$ Institute of Advanced Engineering and Science. All rights reserved.

\section{Corresponding Author:}

Cheng Siong Lim,

Faculty of Electrical Engineering,

Universiti Teknologi Malaysia,

81310 UTM Skudai, Johor, Malaysia.

Email: lcsiong@utm.my

\section{INTRODUCTION}

The efficiency of Emergency Medical Services (EMS) is very important to ensure patients survivability [1-3]. One of the EMS efficiency measurements is ambulance response time (ART). Generally, an ambulances response time can be defined as, the interval from the time the call was received by the EMS provider, to the arrival of the ambulance to the emergency scene [4-7]. A demand point is considered covered if it can be served within a defined time or distance to any of the available facilities; while a demand point farther than the defined time or distance is considered as not covered. An ambulances location is one of the factors that directly affects the ART [8]. As a result, ambulance location model is one of the effective ways to improve ART. Ambulance location models can be categorised into deterministic, probabilistic and dynamic models [9]. One of the earliest deterministic models is Maximal Covering Location Problem (MCLP) which has been introduced by Church and Revelle [10]. Given a fixed number of facilities, MCLP is used to maximise the total coverage with limited resources.

MCLP and its variants are the most widely used location models. In 1984, the reorganisation of EMS in Austin, Texas, using MCLP, saved \$3.4 million of construction cost and \$1.2 million of operating costs annually [11]. MCLP has been used for real life problems to solve hierarchically designed health systems [12-13], congested service systems [14] and bus stop allocations [15]. MCLP is a NP-Hard problem. Various approaches, such as exact method, heuristic and meta-heuristic, can be used to solve a MCLP problem. An exact execution of the method can guarantee the most optimal solution, but may have a longer 
processing time, especially for a larger problem. On the other hand, meta-heuristic method does not guarantee the most optimal solution, though a near optimal solution for a large problem can be achieved with a reasonable amount of time. The evaluated approaches in solving MCLP or its extensions are linear programming and the heuristic method Greedy Adding (GAd) algorithm [10], [16], tabu search [17-18], Lagrangean and Surrogate Relaxations [19], Myopic or Greedy heuristic [20], Heuristic Concentration [21], Genetic Algorithm (GA) [22], and Particle Swarm Optimization (PSO) [23].

Coverage of MCLP is considered unrealistic because the coverage can abruptly change from covered to uncovered after exceeding a defined distance. One of the proposed solutions is applying a variable coverage. Berman et al. [24] reviewed the ideas of using different coverage types. The main purpose of this paper is to compare the results of MCLP to gradual coverage location model (GCLP) in terms of EMS delivery. From the simulations, ART and total travel distance are reported.

In the next section, we review previous related work to GCLP and EMS dynamic simulation. In Section 3, we explain both MCLP and GCLP in detail. The simulation setup is given in Section 4. Results and discussion of the findings are presented in Section 5. Lastly, Section 6 concludes the findings.

\section{PREVIOUS RELATED WORKS}

Church and Roberts [25] are the pioneers who proposed step function to replace covered and uncovered disjunction in a typical coverage model. Berman and Krass [26] introduce Generalized Maximal Covering Location problem which also uses the decreasing step function to allow partial coverage. They also show that their problem is identical to the uncapacitated facility location problem (UFLP).

Berman et al. [27] use the coverage decay function to replace the step function. The coverage decay function has two radii Rmin and Rmax where Rmin $\leq$ Rmax. For demands within radius Rmin, it is considered as fully covered. The coverage value between radii Rmin and Rmax is determined by the coverage decay function, $\mathrm{f}$ (rij). Lastly for demands with distance more than radius Rmax, it is considered as uncovered. One of the findings shows that, the proposed model can generalise the m-median and MCLP problems. The location problem solved can also be formulated as UFLP. Karasakal and Karasakal [28] use similar functions to Berman's to investigate gradual coverage and solve it using Lagrangean Relaxation. Drezner et al. [29] explore gradual coverage using branch and bound algorithm. Most of the reviewed researches use MCLP for gradual cover model, but Eiselt and Marianov [30] look into gradual cover model by using Location Set Covering Model (LSCM).

Simulation is used for different reasons in the study of EMS [31]. Swoveland et al. [32] use simulation to find system characteristics between different EMS rules or policies. The output of the simulation is then used to find the most optimal location model. Simulation is also used to test or analyze the effectiveness of new systems or location models [33-34]. Most of the research involving location models focuses on either the coverage of a model, or the efficiency of the algorithm in solving a location model. However, there is limited number of research that validates the coverage of an ambulance location model, with its actual coverage data during EMS delivery.

There has been limited work on dynamic simulation of EMS delivery. Maxwell et al. [35] simulate EMS operations to evaluate the performance of high quality redeployment policies using approximate dynamic programming (ADP). The simulation is based on discrete-events that cover the entire cycle of an emergency call. In the paper, expensive computation has to be calculated in advance in order to lighten the computational load during real time operation. In their EMS delivery simulation, the ambulance redeployment suggested by ADP can be ignored by dispatchers if they choose to. Lim et al. [36] performed an EMS simulation to compare between different ambulance dispatch policies. Lim et al. proposed freeambulance-exploit dispatch which reassigns ambulances that have just served an emergency call. The simulation shows that reroute-enabled dispatch enhances response time for urgent calls.

Henderson and Mason [37] developed BARTSIM which is used as a decision support tool for ambulance managers at St. John Ambulance Service in the Auckland Region of New Zealand. BARTSIM has a number of important features such as the time travel model, direct reuse of recorded data and spatial visualisation of historical data and result simulation. BARTSIM pre-computes the shortest path to reduce the computation load during simulation. The authors also discuss concerns that should be considered in a discrete event simulator. 


\section{LOCATION PROBLEM FORMULATION}

\subsection{MCLP}

MCLP model is defined as graph $G=(V \mathrm{U} W, E)$, where $V$ and $W$ represent demand points and potential ambulance sites respectively. E stands for distance between $V$ and $W$. MCLP model is written as follows:

$$
\begin{array}{ll}
\text { Maximize } \sum_{i \in V} d_{i} y_{i} & \\
\sum_{j \in W_{i}} x_{i} \geq y_{i} & i \in V \\
\sum_{j \in W_{i}} x_{j}=p & \\
x_{j} \in\{0,1\} & j \in W \\
y_{i} \in\{0,1\} & i \in V
\end{array}
$$

$d_{i}$ represents the demand at point $i . y_{i}$ is a binary variable that will be 1 if and only if point $i$ is covered by at least one ambulance. $x_{j}$ is binary variable that will be 1 if an ambulance is allocated at site $j . p$ is the number of ambulances to be located.

\subsection{GCLP}

For each demand point $i \in V$, there exists two radii $R_{\max }$ and $R_{\min }$ where $0<R_{\min }<R_{\max } . r_{i j}$ is the shortest travel distance from ambulance site $j$ to demand point $i$. A demand point is considered as fully covered if its distance to the closest ambulance site is, $\mathrm{r}_{\mathrm{ij}}<R_{\text {min }}$. Otherwise, it is either deemed as partially covered if $R_{\min }<r_{i j}<R_{\max }$, or uncovered if $r_{i j}>R_{\max }$. In this paper, the following decay function is used. If an area is covered by multiple ambulances, the ambulance with the highest coverage value is chosen. Note that if $R_{\min }$ is equal to $R_{\max }$, GCLP becomes identical to MCLP model. The coverage decay function $\mathrm{f}\left(\mathrm{r}_{\mathrm{ij}}\right)$ is defined as:

$$
f\left(r_{i j}\right)=\left\{\begin{array}{lll}
1 & \text { if } & r_{i j} \leq R_{\min } \\
\frac{\left(R_{\max }-r_{i j}\right)}{R_{\max }-R_{\min }} & \text { if } & R_{\min }<r_{i j} \leq R_{\max } \\
0 & \text { if } & r_{i j}>R_{\max }
\end{array}\right.
$$

Finally, the gradual cover model is defined as follows:

$$
\begin{array}{ll}
\text { Maximize } \sum_{i \in V} d_{i} f\left(r_{i j}\right) & \forall j \in W \\
\sum_{j \in W_{i}} x_{j} \geq f\left(r_{i j}\right) & \forall i \in V \\
\sum_{j \in W} x_{j}=p & \\
x_{j} \in\{0,1\} & j \in W
\end{array}
$$

where all parameters are defined similar to the MCLP algorithm.

\section{METHODOLOGY}

There are a number of methods that have been proposed to solve an ambulance location model such as exact method, mathematical programming heuristic and meta-heuristic methods. The exact method can be 
used to find all the possible combinations of facilities and identify the combination with the best objective function. However, solving an ambulance location model by using the exact method can take a very long time if the value of $\mathrm{W}$ and $\mathrm{V}$ are large. The second method uses mathematical programming. However, commercial mathematical programming software may also fail to solve larger problems [28]. By using heuristic or meta-heuristic method, near optimal results can be produced, but at a shorter time compared to the exact and mathematical programming methods. We have chosen to use PSO to find strategic ambulance locations because it has been successfully proven to solve numerous combinatorial problems [38].

Due to a lack of real data and EMS research [39], EMS demand is generated based on the population of the Johor Bahru region. The region is split into demand zones with the size of $42 \mathrm{~km} \mathrm{x} 30 \mathrm{~km}$ and divided by rectangular grids. The population data is mapped as demands in the region so as to reflect the real Johor Bahru population. A total of 1212 annual demands, which are proportional to the population density, are plotted. In the region, there are 4 hospitals, and 55 potential ambulance sites based at the petrol stations. For simplicity, only one type of ambulances is used. There is no turnout time for the ambulances. Other attributes that may affect the velocity of ambulances such as the type of roads, traffic conditions and traffic lights are ignored. Euclidian distance is used for distance measurement and the calculation of ambulance travel paths.

The simulation is based on a complete cycle of an emergency call, which consists of; (1) acceptance of an emergency call, (2) ambulance dispatch to the emergency scene, (3) paramedics treatment on the victim, (4) transportation of the victim to the hospital, (5) transfer of victim in the hospital, and (6) return of the ambulance to its base. Call set is randomly generated based on the population data. We generate 40 incoming calls based on the Johor Bahru population for an 8 hour simulation time. Many factors can contribute to the variance of call priority as discussed in [40-41]. We divided the calls into low priority and high priority with 1:1 ratio. The closest ambulance is always dispatched to the call, and an ambulance on the way to a low priority call can be rerouted to a high priority call. Zones with higher populations have a higher chance of generating emergency calls. Each ambulance has a constant speed of $60 \mathrm{~km} / \mathrm{h}$. For each call, an ambulance is needed and all victims are required to be transported to the hospital. Each ambulance needs a fixed time of 10 minutes to serve a victim at an emergency scene, and another 10 minutes for the victim to be transferred to the hospital.

For each model of MCLP and GCLP, the simulation is performed for nine fleet sizes in the range of four to 12 ambulances. The fleet size less than three ambulances is omitted because of high ART (> 70 minutes). For each model, two different critical distances are used. Firstly, Rmax=10 and Rmin=3.3 are used. For the second case, $R \max =6$ and $\mathrm{Rmin}=2$ are applied. Note that Rmin is only applicable to GCLP. Thus, a total of 36 simulations are performed.

\section{RESULT AND DISCUSSION}

\subsection{Ambulance Strategic Location}

Figures 1 and 2 show the results of the best ambulance locations found for 6 ambulances based on MCLP and GCLP models respectively. The small circles in black are the identified strategic ambulance locations. The radii for small and big circles are Rmin and Rmax respectively. Demands are categorised with different colours, with red denoting the highest demand and then followed by other colours as follows: red > blue > light blue > yellow > green. In Figure 1, MCLP attempts to maximise coverage by covering all demands by circles with radius Rmax. Note that it has covered $100 \%$ of the demands based on MLCP coverage definition. We can deduce from Figure 1 that when extra ambulances are added, hardly any improvement can be achieved because the demands have been considered as fully covered. In other words, the functions in MCLP are incapable of optimising the extra ambulances for further coverage improvement.

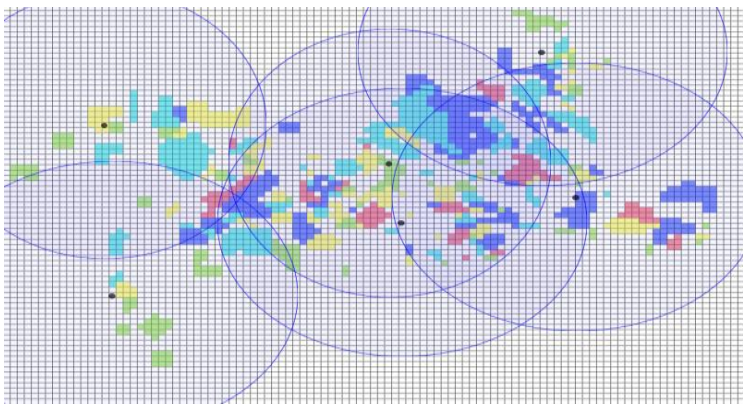

Figure $1 . \mathrm{MCLP}, 100 \%$ covered $(\mathrm{Rmax}=10 \mathrm{~km})$

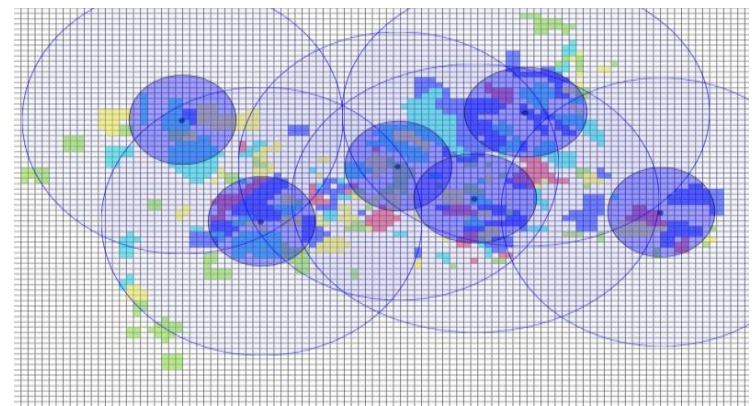

Figure 2 . GCLP, $90.72 \%$ covered $\left(R_{\max }=10 \mathrm{~km}, R_{\min }=3.3 \mathrm{~km}\right.$

IJECE Vol. 7, No. 5, October 2017: $2791-2797$ 


\subsection{Simulation Result}

In Figure 2, GCLP tries to position ambulances as close as possible to the areas with a high number of demands. This is because within Rmin, the area is considered as fully covered, while for the area between Rmin and Rmax, the area is considered as partially covered. As any demand not covered by circles with radius Rmin is considered as not fully covered, there is still room for improvement when extra ambulances are added.

Figure 3 shows that ART for GCLP is shorter than MCLP. One reason for poor results of MCLP is the inability of the model to further improve the coverage with extra ambulances, when it has reached full coverage. For GCLP, further optimisation can still be achieved with extra ambulances because it needs more ambulances to reach full coverage. Figure 4 shows the results with coverage radius, Rmax=6km. GCLP scores lower ART except with a fleet size of four ambulances. In general, GCLP performs better than MCLP even when MCLP has yet to reach full coverage. For $\mathrm{Rmax}=6 \mathrm{~km}$, a fleet size of eight is required for MCLP to reach full coverage. Figures 5 and 6 show the total distance travelled by ambulances of various fleet sizes. As can be seen, the total travel distance can be highly affected by the selection of the ambulance location models. However, for most of the cases, total travel distance for GCLP is lower than MCLP.

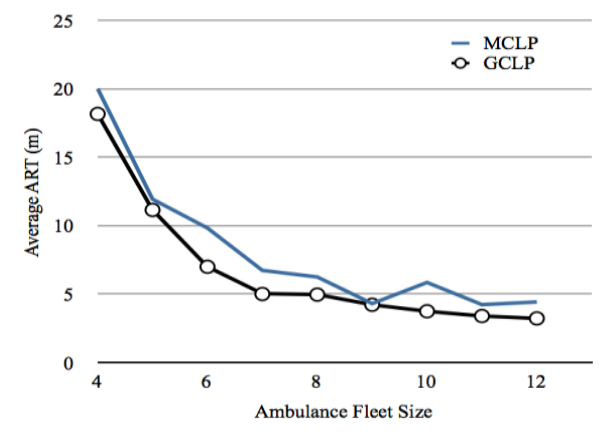

Figure 3. Average ART vs. Ambulance Fleet Size $\left(R_{\max }=10 \mathrm{~km}, R_{\min }=3.3 \mathrm{~km}\right)$

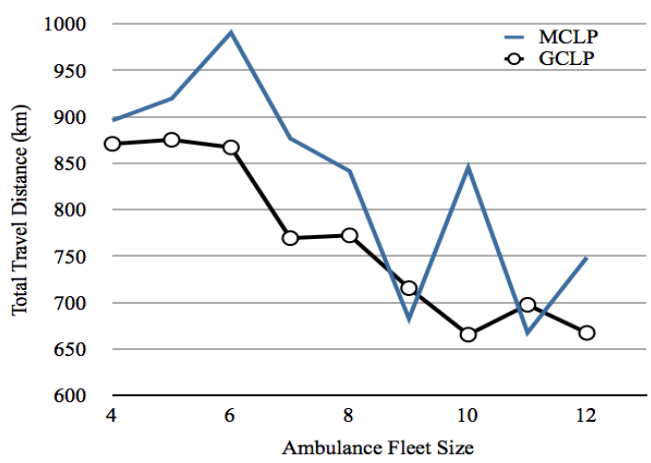

Figure 5. Total Ambulance Travelled Distance vs. Ambulance Fleet Size $\left(R_{\max }=10 \mathrm{~km}, R_{\min }=3.3 \mathrm{~km}\right)$

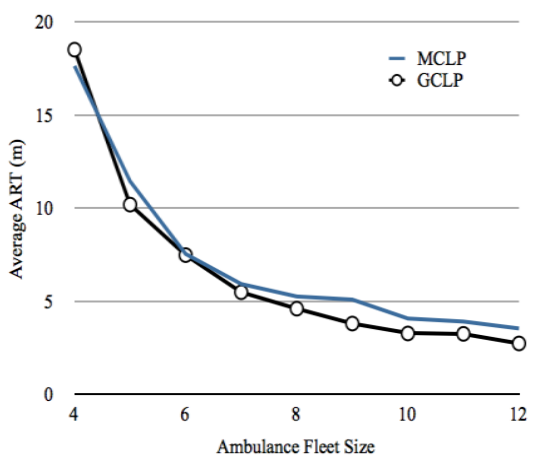

Figure 4. Average ART vs. Ambulance Fleet Size $\left(R_{\max }=6 \mathrm{~km}, R_{\min }=2 \mathrm{~km}\right)$

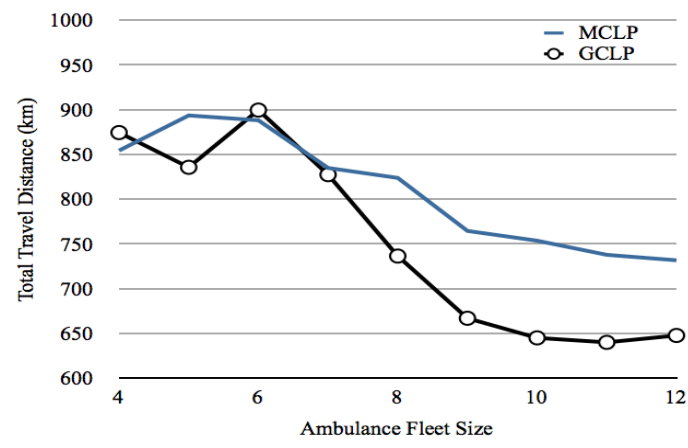

Figure 6. Total Ambulance Travelled Distance vs. Ambulance Fleet Size $\left(R_{\max }=6 \mathrm{~km}, R_{\min }=2 \mathrm{~km}\right)$

\section{CONCLUSION}

There are a number of limitations in this research. The first drawback is the use of Euclidean distance. Actual distance of the road network should be used so that the results can reflect the real life performance. Factors that affect the travel time of ambulances such as road types and traffic conditions must also be considered. If all the above-mentioned proposals are implemented, the ART is expected to be higher than the current simulation results.

Despite the mentioned limitations in the research work, the simulation results have clearly reflected the advantages of GCLP over classical MCLP in EMS delivery. GCLP is capable of optimising extra ambulances in the scenarios which are deemed as fully covered in MCLP. Although the improvement is marginal, any ART improvement in emergency cases would directly increase the survival rate. 


\section{ACKNOWLEDGEMENTS}

The authors would like to acknowledge the financial support from Fundamental Research Grant Scheme (vote no R.J130000.7823.4F314) of the Ministry of Higher Education (MOHE), and Research University Grant (vote on Q.J130000.2523.05H59) from Research Management Centre (RMC) of Universiti Teknologi Malaysia.

\section{REFERENCES}

[1] R. Sánchez-Mangas, A. García-Ferrrer, A. de Juan, and A. M. Arroyo, "The Probability of Death in Road Traffic Accidents. How Important is a Quick Medical Response?” Accid. Anal. Prev., vol. 42, no. 4, pp. 1048-56, Jul. 2010.

[2] R. P. Gonzalez, G. R. Cummings, H. a Phelan, M. S. Mulekar, and C. B. Rodning, "Does Increased Emergency Medical Services Prehospital Time Affect Patient Mortality in Rural Motor Vehicle Crashes? A Statewide Analysis," Am. J. Surg., vol. 197, no. 1, pp. 30-4, Jan. 2009.

[3] R. B. Vukmir, "Survival from Prehospital Cardiac Arrest is Critically Dependent upon Response Time," Resuscitation, vol. 69, no. 2, pp. 229-34, May 2006.

[4] M. Castrén, R. Karlsten, F. Lippert, E. F. Christensen, E. Bovim, a M. Kvam, I. Robertson-Steel, J. Overton, T. Kraft, L. Engerstrom, and L. Garcia-Castrill Riego, "Recommended Guidelines for Reporting on Emergency Medical Dispatch when Conducting Research in Emergency Medicine: the Utstein style.," Resuscitation, vol. 79, no. 2, pp. 193-7, Nov. 2008.

[5] A. K. Marsden, "Getting the Right Ambulance to the Right Patient at the Right Time," Accid. Emerg. Nurs., vol. 3, no. 4, pp. 177-83, Oct. 1995.

[6] U. K. N. Statistics, Ambulance Services England 2008-2009. NHS Information Centre, 2009.

[7] Peter T. Pons and Vincent J. Markovchick, "Eight Minutes Or Less: Does The Ambulance Response Time Guideline Impact Trauma Patient Outcome ?,” J. Emerg. Med., vol. 23, no. 1, pp. 43-48, 2002.

[8] J. J. Bernardo, "Case Study Developing and Validating a Decision Support System for Locating Emergency Medical Vehicles in Louisville, Kentucky,” vol. 75, pp. 567-581, 1994.

[9] L. Brotcorne, G. Laporte, and F. Semet, “Ambulance Location and Relocation Models,” Eur. J. Oper. Res., vol. 147, no. 3, pp. 451-463, Jun. 2003.

[10] R. Church and C. Revelle, "The Maximal Covering Location Problem," vol. 32, pp. 101-118, 1974

[11] D. J. Eaton, M. S. Daskin, D. Simmons, B. Bulloch, G. Jansma, S. Interfaces, C. Tims, P. Papers, J. Feb, J. Eaton, and S. Daskin, "Determining Emergency Medical in Austin , Texas Vehicle Deployment," Interfaces (Providence)., vol. 15, no. 1, pp. 96-108, 1985.

[12] P. Mitropoulos, I. Mitropoulos, I. Giannikos, and A. Sissouras, "A Biobjective Model for the Locational Planning of Hospitals and Health Centers," Health Care Manag. Sci., vol. 9, no. 2, pp. 171-179, Jul. 2006.

[13] G. C. Moore and C. Revelle, "The Hierarchical Service Location Problem,” Manage. Sci., vol. 28, no. 7, pp. 775$780,1982$.

[14] V. Marianov and D. Serra, "Hierarchical Location-allocation Models for Congested Systems," Eur. J. Oper. Res., vol. 135, no. 1, pp. 195-208, Nov. 2001.

[15] J. M. Gleason, “A Set Covering Approach to Bus Stop Location,” Omega, vol. 3, no. 5, pp. 605-608, Oct. 1975.

[16] M. H. Azizan, C. S. Lim, W. A. L. W. M. Hatta, T. L. Go, and S. S. Teoh, "Simulation of Emergency Medical Services Delivery Performance Based on Real Map,” Int. J. Eng. Technol., vol. 5, no. 3, pp. 2620-2627, 2013.

[17] M. Gendreau, "Solving an Ambulance Location Model by Tabu Search," Locat. Sci., vol. 5, no. 2, pp. 75-88, 1997.

[18] J. M. Lee and Y. H. Lee, "Tabu based heuristics for the generalized hierarchical covering location problem," Comput. Ind. Eng., vol. 58, no. 4, pp. 638-645, May 2010.

[19] R. D. Galvao, L. G. A. Espejo, and B. Boffey, "A Comparison of Lagrangean and Surrogate Relaxations for the Maximal Covering Location Problem,” Eur. J. Oper. Res., vol. 124, pp. 377-389, 2000.

[20] M. Daskin, Network and Discrete Location: Models, Algorithms, and Applications. Canada: John Wiley and Sons, 1995.

[21] C. ReVelle, M. Scholssberg, and J. Williams, "Solving the Maximal Covering Location Problem with Heuristic Concentration," Comput. Oper. Res., vol. 35, no. 2, pp. 427-435, Feb. 2008.

[22] M. H. Fazel Zarandi, S. Davari, and S. a. Haddad Sisakht, "The Large Scale Maximal Covering Location Problem," Sci. Iran., vol. 18, no. 6, pp. 1564-1570, Dec. 2011.

[23] W. A. L. W. M. Hatta, C. S. Lim, A. F. Z. Abidin, M. H. Azizan, and S. S. Teoh, "Solving Maximal Covering Location with Particle Swarm Optimization,” Int. J. Eng. Technol., vol. 5, no. 4, pp. 3301-3306, 2013.

[24] O. Berman, Z. Drezner, and D. Krass, "Generalized coverage: New Developments in Covering Location Models," Comput. Oper. Res., vol. 37, no. 10, pp. 1675-1687, Oct. 2010.

[25] R. Church and K. Roberts, "Generalized Coverage Models and Public Facility Location,” Pap. Reg. Sci. Assoc., vol. 53, no. 1, pp. 117-135, 1983 .

[26] O. Berman and D. Krass, "The Generalized Maximal Covering Location Problem," Comput. Oper. Res., vol. 29, no. 6, pp. 563-581, May 2002.

[27] O. Berman, D. Krass, and Z. Drezner, "The Gradual Covering Decay Location Problem on a Network,” Eur. J. Oper. Res., vol. 151, no. 3, pp. 474-480, Dec. 2003.

[28] O. Karasakal and E. K. Karasakal, "A Maximal Covering Location Model in the Presence of Partial Coverage," Comput. Oper. Res., vol. 31, no. 9, pp. 1515-1526, Aug. 2004 
[29] Z. Drezner, G. O. Wesolowsky, and T. Drezner, “The Gradual Covering Problem,” Nav. Res. Logist., vol. 51, no. 6, pp. 841-855, Sep. 2004.

[30] H. A. Eiselt and V. Marianov, "Gradual Location Set Covering with Service Quality," Socioecon. Plann. Sci., vol. 43, no. 2, pp. 121-130, Jun. 2009.

[31] J. Goldberg, R. Dietrich, J. M. Chen, M. Mitwasi, T. Valenzuela, and E. Criss, "A Simulation Model for Evaluating a Set Of Emergency Vehicle Base Locations: Development, Validation, and Usage,” Socioecon. Plann. Sci., vol. 24, no. 2, pp. 125-141, 1990.

[32] A. C. Swoveland, D. Uyeno, I. Vertinsky, and R. Vickson, "Ambulance Location: A Probabilistic Enumeration Approach," vol. 20, no. 4, pp. 686-698, 2014.

[33] S. I. Harewood, "Emergency Ambulance Deployment in Barbados: a multi-objective approach,” J. Oper. Res. Soc., vol. 53, no. 2, pp. 185-192, Feb. 2002.

[34] S. Ingolfsson, A., Erkut, E., Budge, "Simulation of Single Start Station for Edmonton EMS," J. Oper. Res. Soc., vol. 54, no. 7, pp. 736-746, 2003.

[35] M. S. Maxwell, S. G. Henderson, and H. Topaloglu, "Ambulance Redeployment: An Approximate Dynamic Programming Approach,” Winter Simul. ..., pp. 1850-1860, 2009.

[36] C. S. Lim, R. Mamat, and T. Braunl, "Impact of Ambulance Dispatch Policies on Performance of Emergency Medical Services,” IEEE Trans. Intell. Transp. Syst., vol. 12, no. 2, pp. 624-632, Jun. 2011.

[37] S. G. Henderson and A. J. Mason, "Ambulance Service Planning: Simulation and Data Visualisation,” in Operations Research and Health Care, W. P. Brandeau, M. L., Sainfort, F. and Pierskalla, Ed. Springer US, 2005, pp. 77-102.

[38] A. Ghaderi, M. S. Jabalameli, F. Barzinpour, and R. Rahmaniani, "An Efficient Hybrid Particle Swarm Optimization Algorithm for Solving the Uncapacitated Continuous Location-Allocation Problem," Networks Spat. Econ., vol. 12, no. 3, pp. 421-439, Jul. 2011.

[39] T. L. Go, C. S. Lim, K. A. Danapalasingam, M. Loong, P. Tan, and C. W. Tan, "Jurnal Teknologi Full paper A Review on Development and Optimization of Emergency Medical Services in Malaysia," vol. 3, pp. 93-96, 2014.

[40] L. M. Beillon, B.-O. Suserud, I. Karlberg, and J. Herlitz, "Does Ambulance Use Differ Between Geographic Areas? A Survey of Ambulance Use in Sparsely and Densely Populated Areas," Am. J. Emerg. Med., vol. 27, no. 2, pp. 202-11, Mar. 2009.

[41] A. Khorram-Manesh, K. M. Lennquist, A. Hedelin, M. Kihlgren, and P. Örtenwall, "Prehospital Triage, Discrepancy in Priority-setting Between Emergency Medical Dispatch Centre and Ambulance Crews," Eur. J. Trauma Emerg. Surg., vol. 37, no. 1, pp. 73-78, May 2010. 